\title{
Pulmonary innate lymphoid cells are major producers of IL-5 and IL-13 in murine models of allergic asthma
}

\author{
Roel G. J. Klein Wolterink ${ }^{1,2,3,4}$, Alex KleinJan ${ }^{1}$, Menno van Nimwegen ${ }^{1}$, \\ Ingrid Bergen $^{1}$, Marjolein de Bruijn ${ }^{1}$, Yelvi Levani ${ }^{1}$ and Rudi W. Hendriks ${ }^{1}$ \\ ${ }^{1}$ Department of Pulmonary Medicine, Erasmus MC, Rotterdam, The Netherlands \\ ${ }^{2}$ Innate Immunity Unit Department of Immunology, Institut Pasteur, Paris, France \\ ${ }^{3}$ INSERM U668, Institut Pasteur, Paris, France \\ ${ }^{4}$ University of Paris Diderot, Sorbonne Paris Cité, Paris, France
}

Allergic asthma is characterized by chronic airway inflammation and hyperreactivity and is thought to be mediated by an adaptive T helper-2 (Th2) cell-type immune response. Here, we demonstrate that type 2 pulmonary innate lymphoid cells (ILC2s) significantly contribute to production of the key cytokines IL-5 and IL-13 in experimental asthma. In naive mice, lineage-marker negative ILC2s expressing IL-7R $\alpha$, CD25, Sca-1, and T1/ST2(IL-33R) were present in lungs and mediastinal lymph nodes (MedLNs), but not in broncho-alveolar lavage (BAL) fluid. Upon intranasal administration of IL-25 or IL-33, an asthma phenotype was induced, whereby ILC2s accumulated in lungs, MedLNs, and BAL fluid. After IL-25 and IL-33 administration, ILC2s constituted $~ 50$ and $\sim 80 \%$ of IL- $5^{+} / \mathrm{IL}-13^{+}$cells in lung and BAL, respectively. Also in house dust mite-induced or ovalbumin-induced allergic asthma, the ILC2 population in lung and BAL fluid increased significantly in size and ILC2s were a major source of IL-5 or IL-13. Particularly in OVAinduced asthma, the contribution of ILC2s to the total population of intracellular IL-5+ and IL- $13^{+}$cells in the lung was in the same range as found for Th2 cells. We conclude that both ILC2s and Th2 cells produce large amounts of IL-5 and IL-13 that contribute to allergic airway inflammation.

Keywords: Asthma $•$ Cytokines $•$ House dust mite $\bullet$ Innate immunity $\bullet$ Innate lymphoid cells

See accompanying Commentary by Spits

Supporting Information available online

\section{Introduction}

Allergic asthma is characterized by a predominant eosinophilic airway inflammation, airway hyperreactivity, and a chronic $\mathrm{T}$ helper-2 (Th2) cell-type of immune response to various allergens, such as house dust mites (HDMs), molds, or animal dander [1,2]. Available experimental models for asthma indicate that allergenspecific Th2 cells are key players in induction and maintenance of allergic asthma [3]. These cells produce vast amounts of cytokines

Correspondence: Dr. Rudi W. Hendriks

e-mail: r.hendriks@erasmusmc.nl that induce IgE synthesis (IL-4), recruit eosinophils and mast cells (via IL-5 and IL-9, respectively), and cause smooth muscle hyperreactivity via IL-13 (reviewed in [4]).

Although adaptive Th2 cells have been identified as important sources of IL-4, IL-5, and IL-13 [5], many recent studies emphasize the importance of innate cells in cytokine production, including but not limited to mast cells, eosinophils, and basophils. Next to the canonical NK and lymphoid-tissue inducer (LTi) cells, a growing family of cytokine-producing "helper" innate lymphoid cells (ILCs) has been identified [6]. Interestingly, a lineage-negative ILC population that produces high amounts of IL-5 and IL-13 was identified in fat-associated lymphoid clusters (FALCs) and 
mesenteric lymph nodes [7-9]. These cells were named natural helper cells, nuocytes, or multipotent progenitors and, because of their Th2 cytokine production, they have been dubbed type 2 ILCs [6]. ILC2s play a role in defense mechanisms and (re)shaping immune and nonimmune tissues and can be stimulated with IL-25 and IL-33. In a Nocardia brasiliensis infection model, ILC2s were essential and sufficient for clearance of this helminth from the mucosa, even in the absence of the adaptive immune system [7,9].

Recently, various groups demonstrated the presence of ILC2s in the respiratory system of mice and humans [10-12]. ILC2 were shown to accumulate in the lungs of mice after infection with influenza virus, via an IL-33 dependent mechanism. These ILC2 induced airway hyperreactivity through IL-13 secretion [10] and also restored airway epithelial integrity and lung function and contributed to airway remodeling by the production of amphiregulin [12]. In addition, ILC2s were found in nasal polyps of patients with chronic rhinitis, a classical Th2 disease [11].

IL-25 is a member of the IL-17 cytokine family that is expressed in human and mouse in response to allergens, particles, and helminth infection [13-15]. Administration of IL-25 was shown to induce IL-4, IL-5, and IL-13 production, even in the absence of $\mathrm{B}$ and $\mathrm{T}$ lymphocytes in recombination activating gene (Rag)deficient mice [15]. The main sources of IL-25 in asthma are the lung epithelium, eosinophils, mast cells, and basophils [16]. Moreover, IL-25 amplifies Th2 responses and causes increased eosinophilic infiltration in mice [17]. IL-33 belongs to the IL-1 family and binds a receptor complex consisting of the IL-1R accessory protein and ST2 [18]. The membrane-bound form of ST2 is highly expressed on mast cells and Th2 cells $[19,20]$, associating the IL-33 pathway with Th2 conditions. IL-33 is produced by epithelial cells in the lung [18] and epithelial expression of IL-33 increases in asthmatics [21]. The importance of IL-25 and IL-33 for asthma is underlined by the finding that disruption of the IL- 25 or IL-33 pathway prevents induction of allergic airway inflammation in mice [22,23]. Several studies have identified lineage-negative cells in the lung that manifest IL- 5 and IL-13 production in response to IL-25 $[14,24]$ or IL-33 $[25,26]$ administration in mice.

In this report, we investigated the presence of ILC2s in the respiratory tract and hypothesized that pulmonary ILC2s are involved in allergic asthma by production of Th2 cytokines. We found that ILC2s produced substantial amounts of IL-5 and IL-13 in various murine models of allergic lung inflammation, both upon intranasal administration of IL-25 or IL-33 and in house dustmite (HDM) or ovalbumin (OVA)-induced asthma. We conclude that both ILC2s and Th2 cells produce large amounts of IL-5 and IL-13 that contribute to allergic inflammation in asthma.

\section{Results}

\section{Identification and characterization of pulmonary ILC2s}

To explore the presence of ILC2s in the respiratory tract, we used flow cytometry and analyzed lung, mediastinal lymph node (MedLN), broncho-alveolar lavage (BAL), and thoracic fat of naive wild-type mice. We identified a lineage-negative, low side scatter population expressing IL-7R $\alpha$ (CD127), T1/ST2 (IL-1RL1, a subunit of IL-33R), partially positive for c-Kit (CD117), stem cell antigen Sca-1 (Ly6A/E), and IL-2R $\alpha$ (CD25) in the lung (Fig. 1A). This population resembled the recently identified population of ILC2s in gut-associated tissue and influenza infected lungs $[6,7,9,10,12]$. Sca-1 expression levels were higher in B6 mice than in BALB/c mice (data not shown). As ILC2s were initially found in fat-associated lymphoid tissue, we analyzed the thoracic fat attached to the thorax wall and around the aorta, and found varying but steadily limited numbers $(<200)$ of $\mathrm{Lin}^{-} \mathrm{T} 1 / \mathrm{ST} 2{ }^{+} \mathrm{CD} 127^{+}{ }^{\mathrm{c}-\mathrm{Kit}^{+}}$cells. These ILC2s were present in the lung and MedLNs of naive mice, but virtually absent in BAL (Fig. 1B).

\section{Pulmonary ILC2s depend on common gamma chain cytokine signals}

Pulmonary ILC2s were present in the lungs of C57BL/6 and BALB/c mice (Supporting Information Fig. 1). Intestinal ILC2s are found in Rag2 $2^{-/-}$mice, but their development or survival depends on the common gamma $\left(\gamma_{c}\right)$ chain, as Rag2/Il2rg double-deficient mice lack ILC2 [7]. Accordingly, we found pulmonary ILC2s in comparable numbers in the lungs and MedLNs of Rag1 $1^{-/-}$mice, but not in Rag2/Il2rg double-deficient mice (Supporting Information Fig. 1). We noticed that the absence of Myd88, an adapter molecule that functions downstream of TLRs and IL-33R [27], had no significant effect on ILC2 numbers in the lung. IL-22 is an important cytokine in the lung and in the intestine for protection of epithelial surfaces [28] and is rapidly produced in conjunction with IL-17 by LTi cells. We detected no significant alterations in lung ILC2 numbers in Il22 $2^{-/-}$mice (Supporting Information Fig. 1). Closely related ILC populations, including ILC17 [29] and ILC22 [30-33], depend on the ROR $\gamma \mathrm{t}$ transcription factor for their development, while intestinal ILC2s do not express ROR $\gamma t$, but $\operatorname{ROR} \alpha$ and $\operatorname{ROR} \beta$ instead [7]. In agreement with this finding, pulmonary ILC2s were present in mice deficient for the Rorc gene, encoding ROR $\gamma \mathrm{t}$ (Supporting Information Fig. 1).

In summary, we found that development of pulmonary ILC2s requires signaling via a $\gamma_{\mathrm{c}}$-dependent cytokine pathway. Because of the high levels of IL-7R $\alpha$ (CD127), it is conceivable that IL-7 signals are important for ILC2 development.

\section{Pulmonary ILC2s produce IL-5 and IL-13 Th2 cytokines}

ILC2s produce large amounts of the Th2 cytokines IL-5 and IL-13 upon phorbol myristate acetate (PMA) and ionomycin stimulation [7,9]. When we stimulated lung cell suspensions with PMA/ionomycin for $4 \mathrm{~h}$ and probed for production of the Th2 cytokines by intracellular flow cytometry, we detected fractions of ILC2s positive for IL-5 and IL-13, while IFN- $\gamma^{+}$and IL- $4^{+}$ILC2s were hardly detectable (Fig. 1C). Quantitative RT-PCR analysis of sorted lin $^{-} \mathrm{CD}_{12} 7^{+} \mathrm{T} 1 / \mathrm{ST} 2^{+}$cells from naive mice stimulated in 

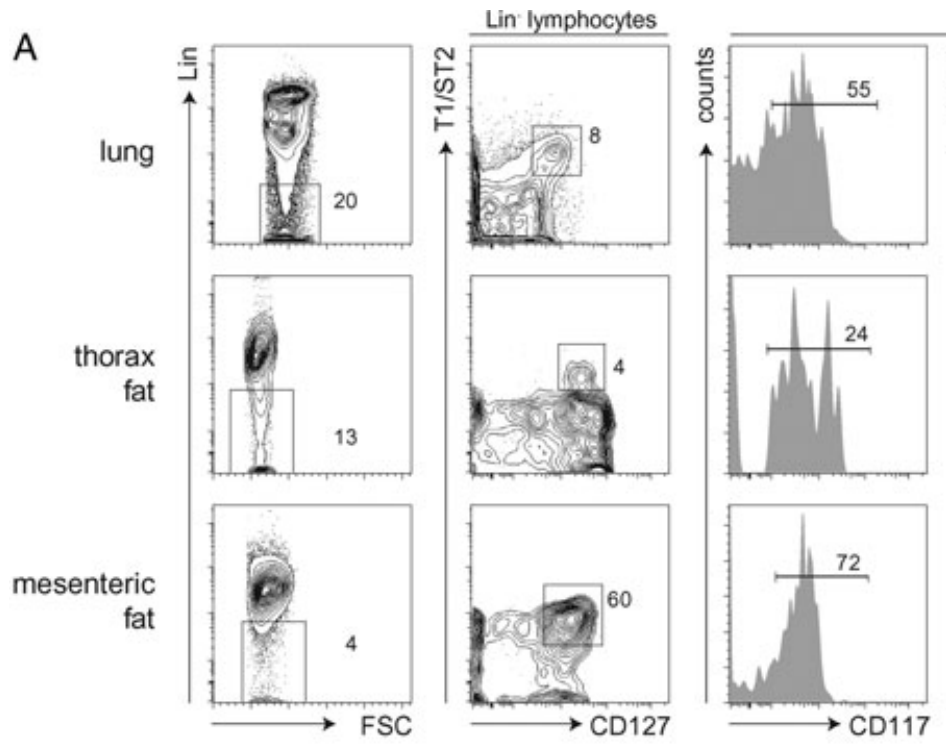

Lin- T1/ST2* CD127*
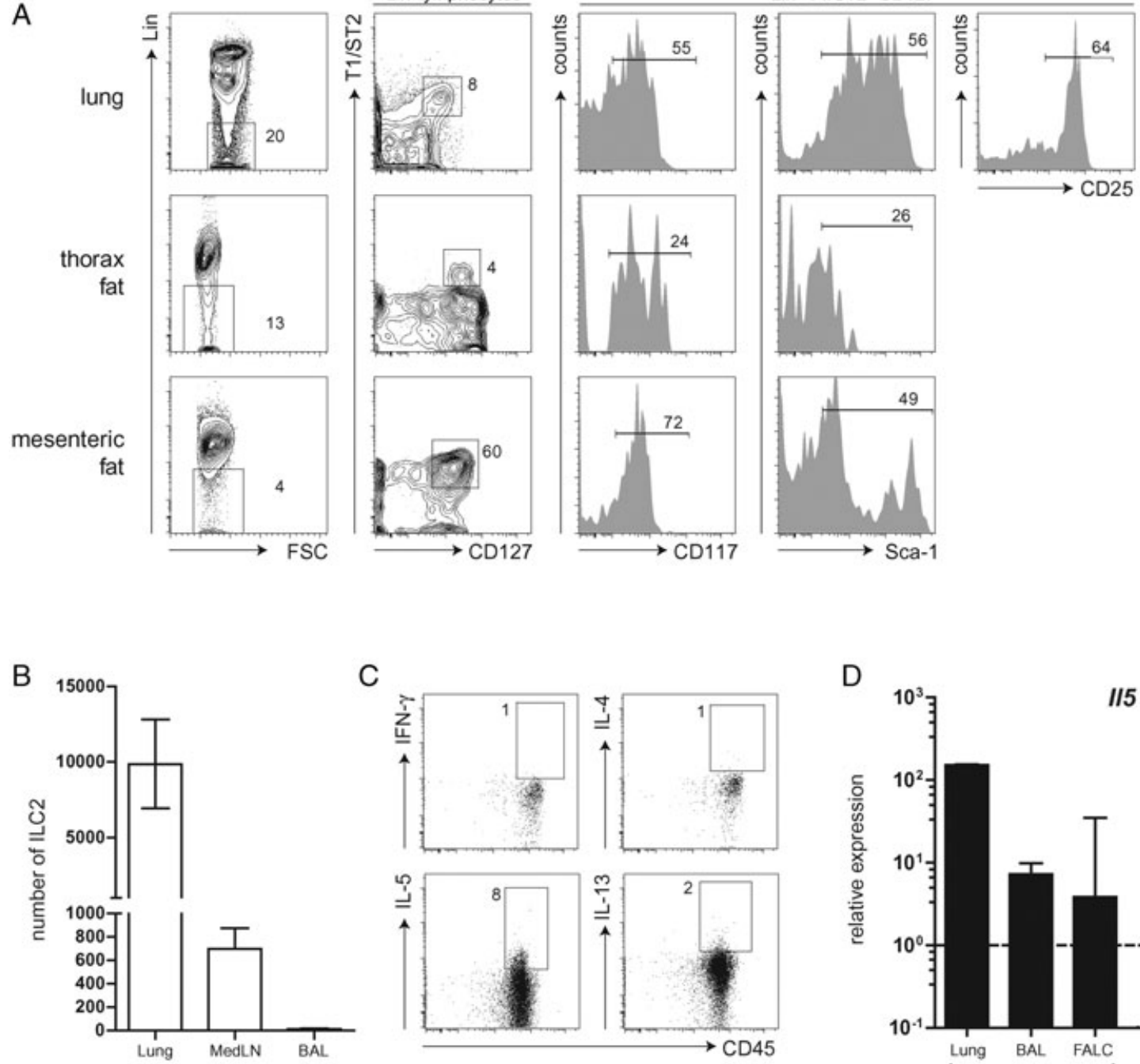

C
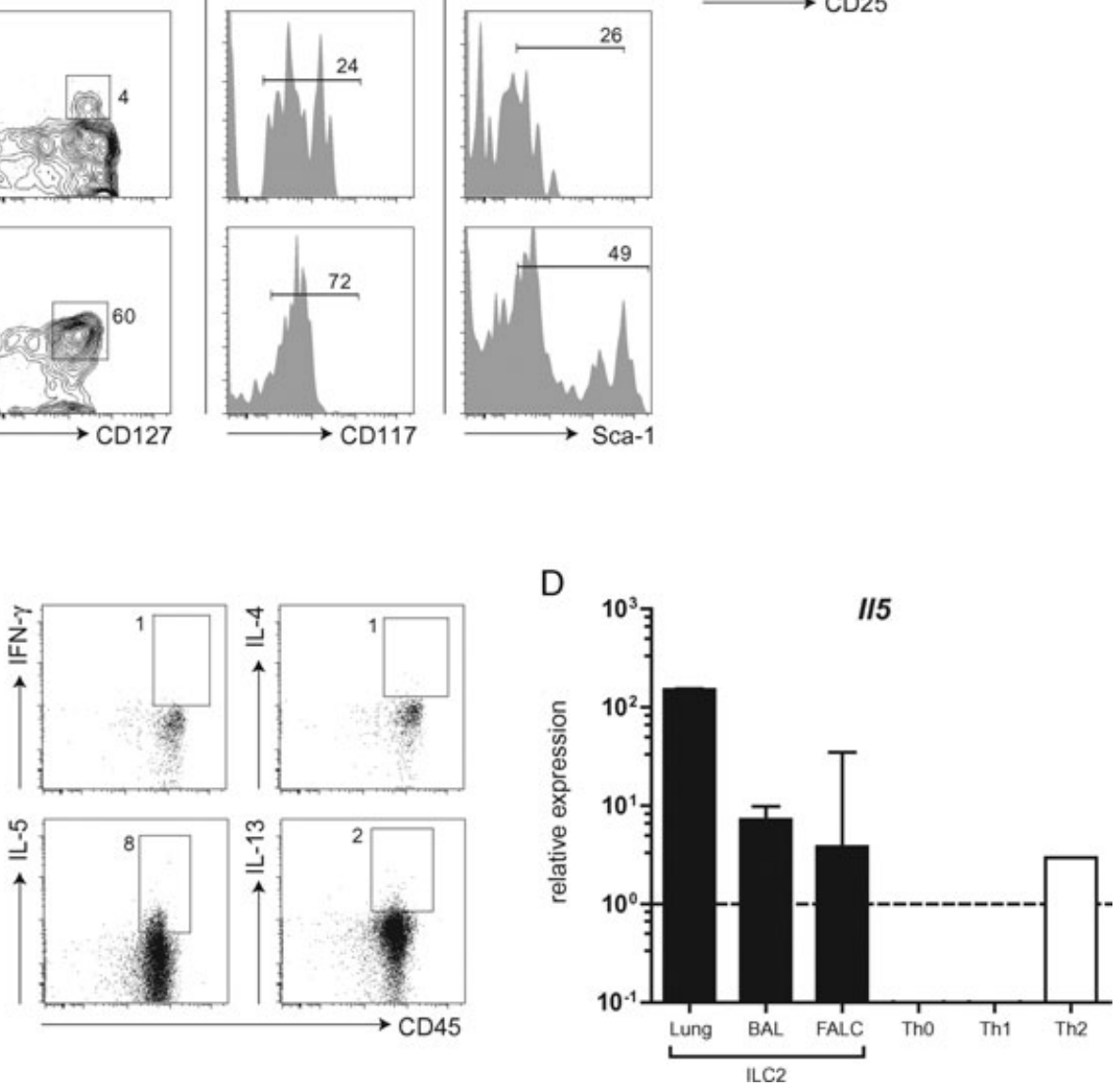

Figure 1. Pulmonary ILC2s are present in the respiratory tract of naive mice. (A) Flow cytometric identification of pulmonary ILC2s is shown. Single-cell suspensions of collagen-treated lungs, thorax fat, and mesenteric fat of naive BALB/c mice were analyzed by flow cytometry. Cells negative for lineage markers were analyzed for expression of IL-33R (T1/ST2) and CD127 (IL-7R $\alpha$ ). T1/ST2+ IL-7R $\alpha^{+}$cells were gated and analyzed for c-Kit (CD117), Sca-1, and IL-2R $\alpha$ (CD25), shown as histograms. Proportions of cells within the gates are given. FSC: Forward Scatter. (B) The quantification of ILC2s ( $\mathrm{Lin}^{-} \mathrm{CD} 45^{+} \mathrm{CD} 127^{+} \mathrm{T} 1 / \mathrm{ST} 2^{+} \mathrm{CD} 25^{+}$cells) in the pulmonary tract is shown. MedLN: mediastinal lymph node; BAL: bronchoalveolar lavage. Data are shown as mean + SEM of $n \geq 2$ animals per group and all data are representative of four independent experiments. (C) Intracellular cytokine content of ILC2s is shown. Lin-CD127 ${ }^{+} \mathrm{T} 1 / \mathrm{ST} 2^{+}$lymphocytes were gated and analyzed for CD45 versus the indicated cytokines. Results are shown as dot plots. Single-cell suspensions were stimulated with PMA/ionomycin in the presence of monensin for $4 \mathrm{~h}$. (D) RT-PCR quantification of Il5 expression of the indicated FACS-sorted ILC2 cell suspensions, as well as polarized T-cell cultured under Th0, Th1, and Th2 conditions is shown. Il5 levels were normalized to Actb levels. Data are shown as mean + SEM of $n \geq 3$ samples per group for ILC2 populations and one sample per group for Th populations and data are representative of two independent experiments.

vitro with IL-2, IL-25, and IL-33 showed that lung ILC2 expressed higher levels of Il5 than BAL ILC2, FALC ILC2, or in vitro cultured polarized Th2 cells (Fig. 1D).

\section{Pulmonary ILC2s are induced in IL-25- and IL-33-induced asthma}

Although classic animal models of experimental asthma depend on sensitized Th2 cells, intranasal administration of IL-25 or IL-33 in mice have also been reported to induce an asthma phenotype with airway hyperresponsiveness, eosinophilic inflammation, mucus hypersecretion, and Th2 cytokine production in the lung $[14,25,34]$. Since ILC2s are reported to release cytokines in response to IL-25 or IL-33 stimulation [7,9], we hypothesized that pulmonary ILC2s contribute to airway inflammation in cytokineinduced asthma. Mice were intranasally treated with five doses of IL-25 or three doses of IL-33 and analyzed $24 \mathrm{~h}$ after the last instillation (Fig. 2A). While we observed no significant increase in BAL cellularity in IL-25 treated mice, IL-33 induced a strong increase in total cellularity, eosinophils, and $\mathrm{T}$ cells in BAL fluid (Fig. 2B), indicating the onset of airway inflammation. Likewise, lung cellularity also increased upon IL-33 administration (Fig. 2C). 
A

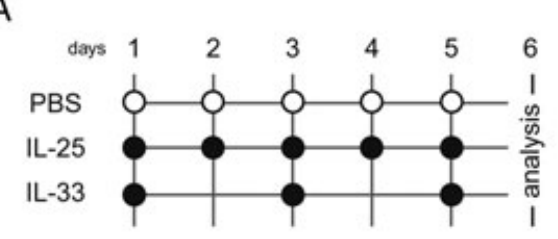

C

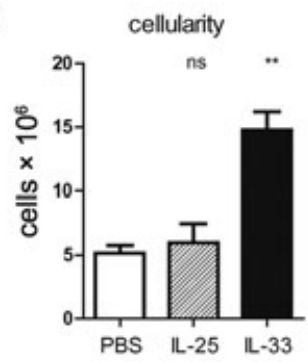

E

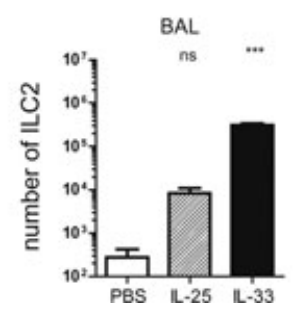

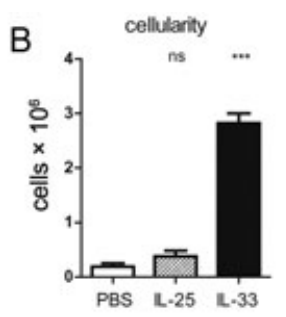
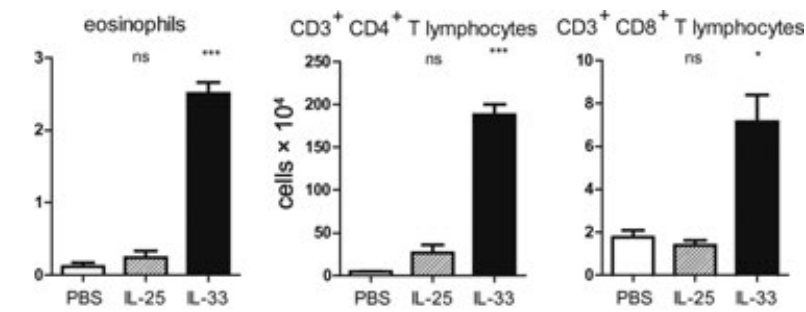

D
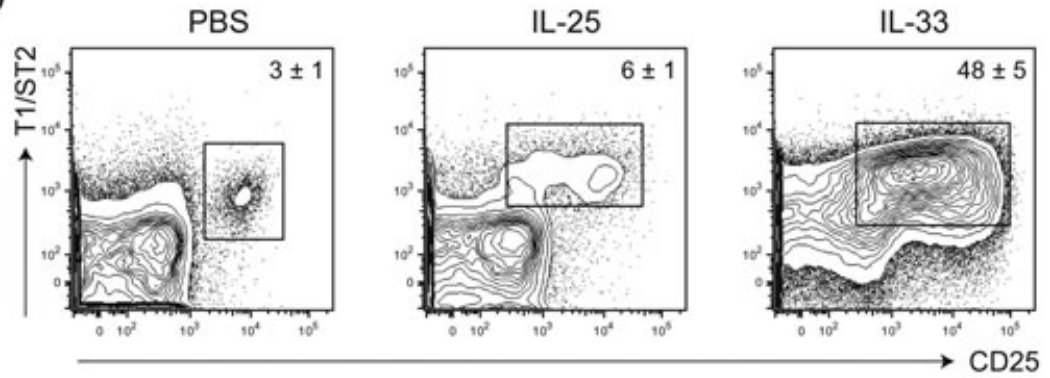

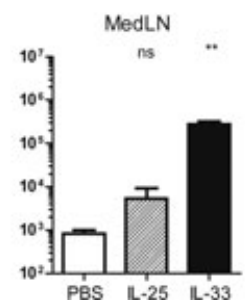

Figure 2. Administration of IL-25 and IL-33 induces eosinophilic airway inflammation and increases ILC2 numbers. (A) Scheme for IL-25 and IL-33 treatment of BALB/C mice. Isoflurane-anesthesized animals received $1 \mu \mathrm{g}$ intranasal administrations of the indicated cytokines (or PBS as a control) on the days marked with a circle and were analyzed $24 \mathrm{~h}$ later. (B) The quantification of indicated cell populations in the BAL fluid after cytokine treatment is shown. (C) The total number of cells in the lungs of treated animals is shown. (D) Flow cytometric identification of pulmonary ILC2s (gated) in single-cell suspensions of the lungs of treated mice. Plots show expression of the indicated markers on cells negative for lineage markers. Numbers indicate the frequency of ILC2s in the total lymphocyte population \pm SEM using the gates indicated. (E) Quantification of ILC2s in different compartments of the pulmonary tract is shown. (B, C, E) Data are shown as mean + SEM of $n=3-4$ mice per group and all panels are representative of two independent experiments. ${ }^{*} p<0.05,{ }^{* *} p<0.01,{ }^{* * *} p<0.001$, one-way ANOVA analysis followed by Turkey post-tests to analyze statistical significance between PBS control versus treated animals. ns: not significant.

Administration of IL-25 and IL-33 induced upregulation of T1/ST2 on ILC2s (Fig. 2D). The number of ILC2s strongly increased in mice treated with IL-33 (Fig. 2E).

\section{Pulmonary ILC2s are the major cytokine producing lymphocytes in IL-25- and IL-33-induced asthma}

Next, we determined the contribution of ILC2s to production of IL-4, IL-5, and IL-13 in cytokine-induced airway inflammation by intracellular flow cytometry. We observed that intranasal treatment with PBS alone induced increased production of IL-5 and IL-13 (Fig. 3A, compared with naive mice, Fig. 1C). Treatment with IL-25 or IL-33 further increased the fraction of IL-5 and IL- $13^{+}$ILC2s to more than $\sim 80$ and $\sim 60 \%$, respectively, while IL-4 production remained low (Fig. 3A). Comparisons with cytokine content of $\mathrm{T} 1 / \mathrm{ST} 2^{+} \mathrm{CD}^{+}{ }^{+} \mathrm{T}$-cell fractions, which are enriched for Th2 polarized cells [20], and T1/ST2 ${ }^{-}$CD4 ${ }^{+}$T-cell fractions, revealed that the ILC2 population comprised most of the IL- $5^{+}$and IL- $13^{+}$lymphocytes upon treatment with IL-25 and IL-33 (up to $3.4 \times 10^{6} \mathrm{IL}-5^{+}$and $3.1 \times 10^{6} \mathrm{IL}_{-1}-13^{+}$cells/lung after IL-33 treatment, respectively) (Fig. 3B). The contribution of ILC2s to IL-4 production strongly increased upon IL-33 but not upon IL-25 administration. Likewise, IL-25 and IL-33 activated IL-5 and IL-13 production in ILC2s in BAL fluid (Supporting Information Fig. 2A). After treatment with IL-25 and IL-33, ILC2 became the most important producers of Th2 cytokines in the BAL (Supporting Information Fig. 2B).

Taken together, these results show that treatment with IL-25 induced a limited increase in BAL cellularity, whereby pulmonary ILC2s were potent producers of IL-5 and IL-13. In contrast, treatment with IL-33 induced a strong increase in total cellularity, and an increase of the numbers of eosinophils and ILC2s in BAL. These ILC2 manifested a high cytokine content for IL-5, IL-13, and to a lesser extent IL-4, all of which contribute to allergic lung inflammation.

\section{ILC2 population is an important target of IL-25 and IL-33, independent of the adaptive immune system}

Previous studies have shown that IL-25 and IL-33 can induce an asthma phenotype, even in the absence of $\mathrm{B}$ and $\mathrm{T}$ lymphocytes 

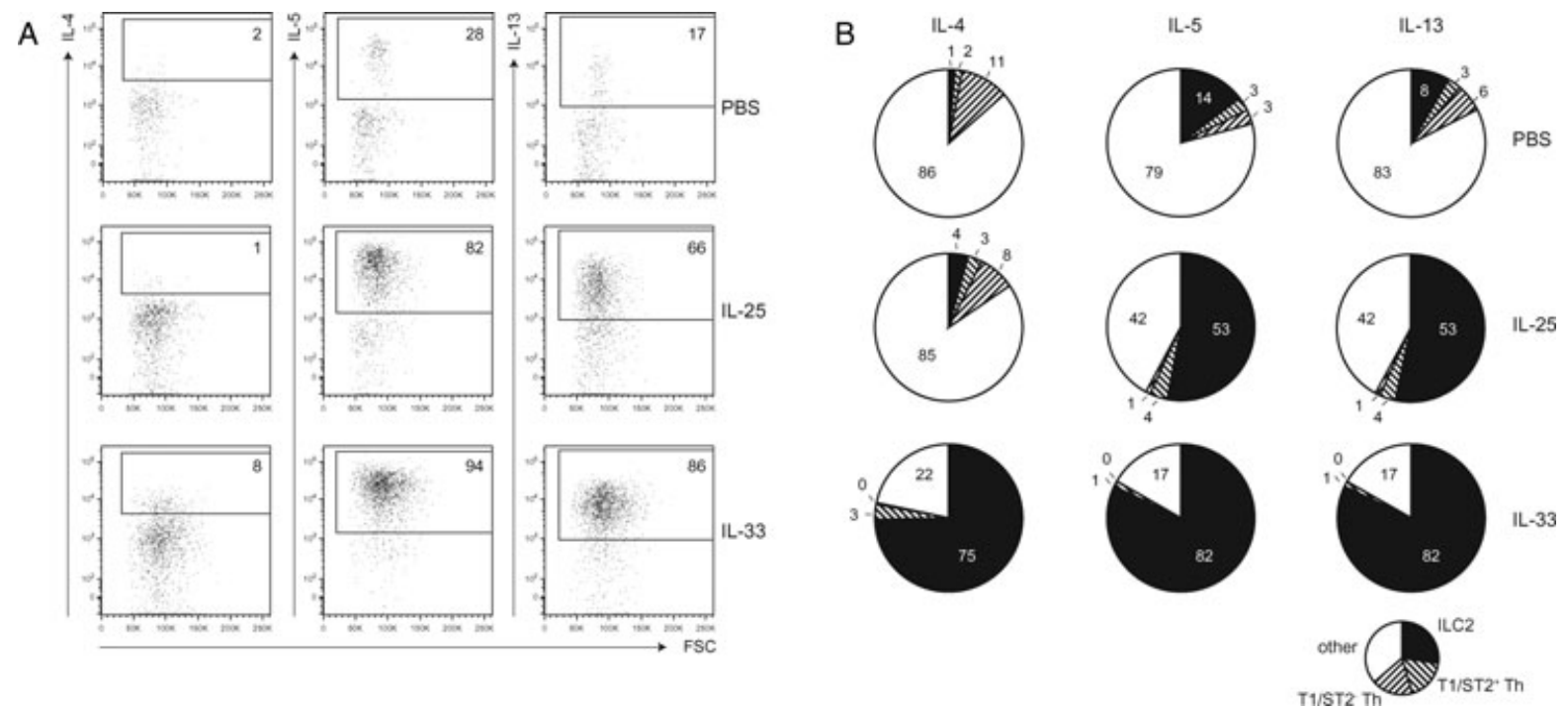

Figure 3. ILC2s produce important amounts of IL-5 and IL-13 in cytokine-induced airway inflammation. (A) Intracellular cytokine content of pulmonary Lin ${ }^{-} \mathrm{CD} 25^{+} \mathrm{CD} 127^{+} \mathrm{T} 1 / \mathrm{ST} 2^{+}$ILC2s in mice, treated as specified. Plots show forward scatter (FSC) versus the indicated cytokines. Singlecell suspensions of lungs were stimulated with PMA/ionomycin in the presence of monensin for $4 \mathrm{~h}$. Numbers indicate the proportions of cytokine-positive ILC2s. (B) Contribution of various cell populations to Th2 cytokine production in the lungs after administration of PBS, IL-25, or IL-33. Proportions of cytokine expressing cells were determined by flow cytometry (as in panel A) in ILC2, T1/ST2 ${ }^{+}$, and T1/ST2- CD3 ${ }^{+}$CD4 ${ }^{+}$ Th cells. Pie charts and percentages represent the contribution of various populations to the total number of cytokine-producing cells. Data are representative of two independent experiments with three to four mice per group.

$[14,25,34]$. Consistent with reported findings [7,8], we found that ILC2s develop in the absence of Rag1 or Rag2 but are virtually absent from lungs in mice that lack the $\gamma \mathrm{c}$ chain (Supporting Information Fig. 1 and 4A). To investigate whether ILC2s are activated in the absence of an adaptive immune system, we employed the cytokine-induced asthma models in Rag2-deficient $\left(\mathrm{ILC}^{+}\right.$) and Rag2/Il2rg double deficient (ILC2 ${ }^{-}$) mice. Administration of IL-25 and IL-33 resulted in an increase in cellularity in lung and BAL and eosinophilic inflammation in Rag2-deficient mice, but not in Rag2/Il2rg double-deficient mice (Fig. 4B). These findings indicate that a $\gamma \mathrm{c}$ chain-dependent population is responsible for inducing airway inflammation upon IL-25/IL-33 administration. Administration of IL-33 induced increased ILC2 numbers in BAL and lung in Rag2-deficient mice, while we observed no increase in ILC2 numbers in Rag/Il2rg-deficient mice (Fig. 4C). Treatment of mice with PBS, IL-25, and IL-33 induced production of IL-5 and IL-13 but not IL-4 in Rag2-deficient lung ILC2 (Supporting Information Fig. 3), demonstrating that ILC2 are activated in the absence of adaptive immunity.

In summary, we found that airway inflammation induced by IL-25 and IL-33 is mediated by a Rag2-independent, $\gamma \mathrm{c}$ chaindependent population. Moreover, a strong increase in the number of ILC2s, more than $\sim 80 \%$ of which were intracellular IL-5 and $\mathrm{IL}-13^{+}$, was induced. Therefore, we conclude that ILC2s are the most important inducers of eosinophilic inflammation upon intranasal IL-25 or IL-33 administration.

\section{Pulmonary ILC2s are important cytokine producers in HDM-induced allergic asthma}

Next, we induced allergic asthma in mice with HDM to determine to which extent ILC2s synergize with an adaptive inflammatory
Th2 response in the respiratory tract. Mice were sensitized with HDM and challenged either once, three, or ten times with HDM (Fig. 5A). Mice that were sensitized and challenged with PBS served as controls. As expected, HDM-challenged mice exhibited airway inflammation marked by increased cellularity, eosinophilia and T-cell influx, and BAL fluid, when compared with PBS controls (Fig. 5B). HDM-induced asthma was also accompanied by increased numbers of ILC2s in lung and BAL, but not in MedLNs (Fig. 5C), indicating that during the Th2 inflammation ILC2s undergo proliferation or are attracted, possibly by cytokines.

As observed in cytokine-induced airway inflammation, in HDMinduced allergic asthma pulmonary ILC2s did not only increase in number, but concomitantly also the proportions of cytokinecontaining cells increased. Intracellular flow cytometry demonstrated that in HDM-induced allergic asthma ILC2s in the lung produced IL-5, IL-13, and also IL-4 (Fig. 5D). To determine to what extent pulmonary ILC2s contribute to cytokine production in relation to conventional Th2 cells and other Th cells, we calculated absolute numbers of cytokine-expressing cells in lungs and BAL. The contribution of ILC2s to IL-4 was limited, compared with $\mathrm{T} 1 / \mathrm{ST}_{2}{ }^{+}$or $\mathrm{T} 1 / \mathrm{ST} 2^{-}$Th cells, and only detectable in the lung, where up to $\sim 1400$ ILC2 produced IL-4. In the lung up to $\sim 1900$ of total IL- $5^{+}$cells were ILC2s, which is substantial, as compared to values between 2000 and 4500 for $\mathrm{T}_{1 / \mathrm{ST}{ }^{+}}$or between 1500 and 4800 for T1/ST2 ${ }^{-}$CD4 ${ }^{+}$T-cell fractions (Fig. 5E). Likewise, up to 2800 ILC2s produced IL-13, while 3200-5800 T1/ST2 ${ }^{+}$Th2 cells produced IL-13. Despite the modest numbers of ILC2s detected in BAL fluid, the number of IL-5 and IL-13 producing ILC2 and $\mathrm{T} / \mathrm{ST}_{2}{ }^{+}$Th2 cells were comparable. Collectively, these results illustrate that pulmonary ILC2s comprised a significant proportion of $\mathrm{IL}^{-} 5^{+}$and $\mathrm{IL}-13^{+}$cells in lung and BAL in HDM-induced allergic asthma, whereby the contribution of ILC2 cells to the total 
A

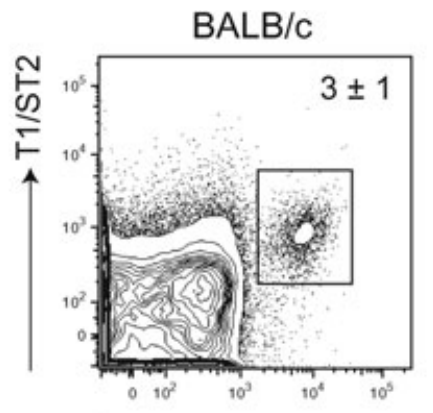

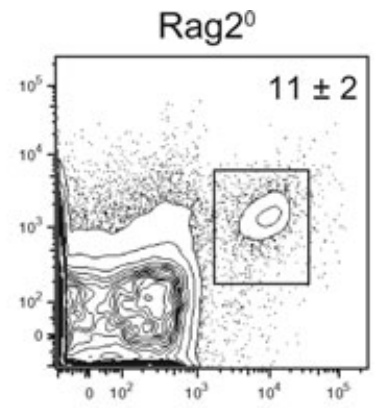

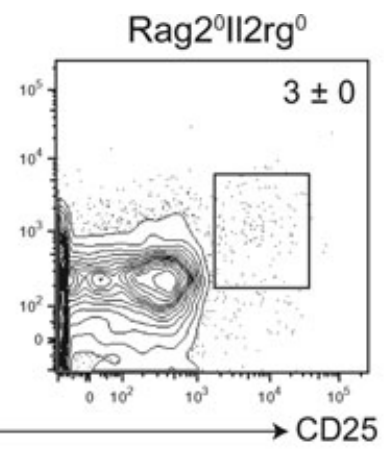

B
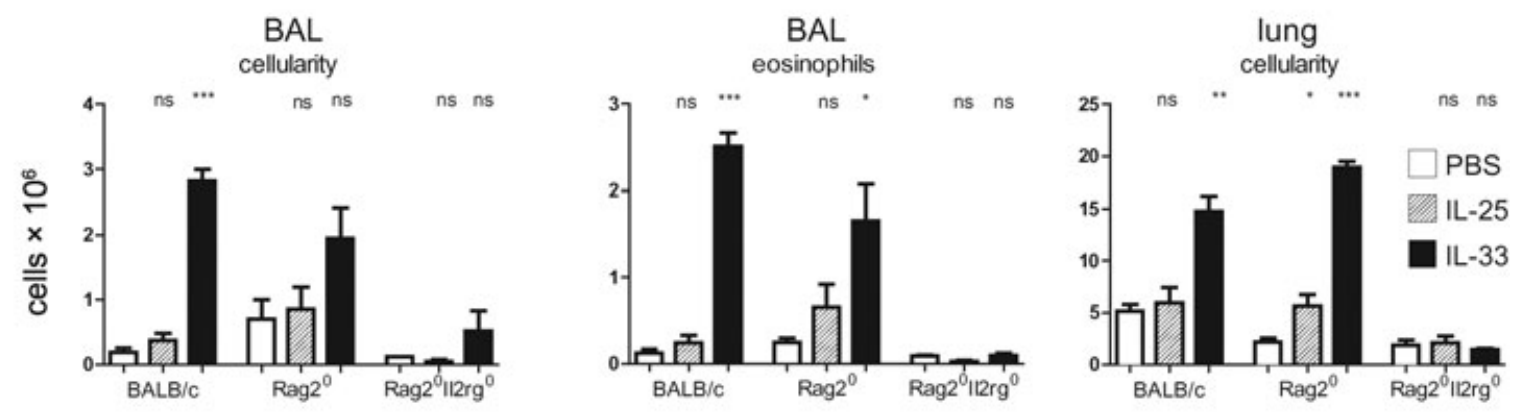

C
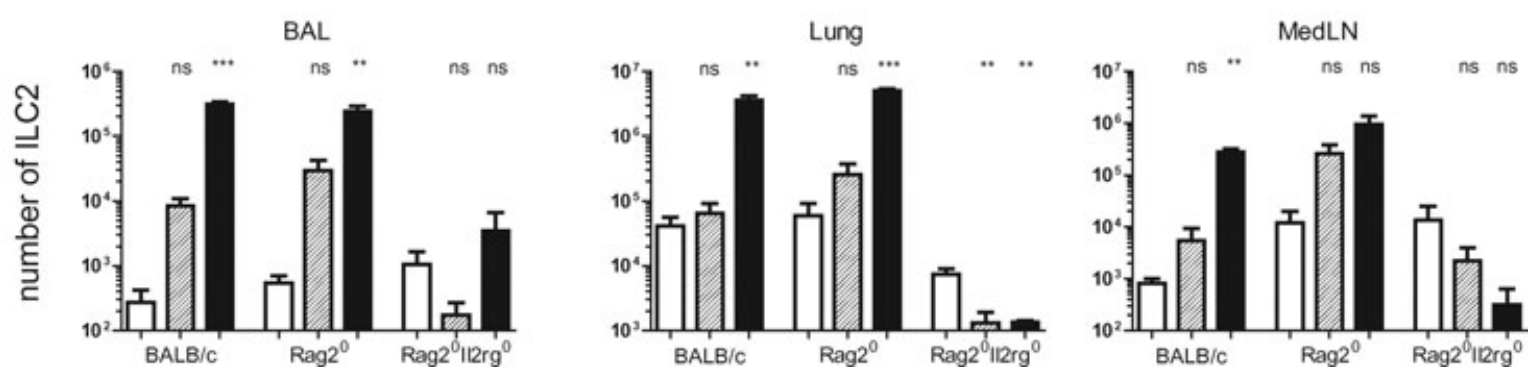

Figure 4. Induction of airway inflammation by IL-25 and IL-33 depends on a $\gamma$ c chain-dependent cell population. (A) Identification of ILC2s in the lungs of mice of the indicated strains is shown. Plots show expression of CD25 and T1/ST2 by Lin ${ }^{-}$CD127 ${ }^{+}$lymphocytes. Values indicate the percentage of ILC2 of Lin-negative lymphocytes \pm SEM. (B) Quantification of indicated cell population in the BAL fluid of the indicated mice treated with PBS, IL-25, or IL-33 in BAL and lung is shown. (C) Quantification of Lin ${ }^{-}$CD $25^{+}$CD $127^{+}$T1/ST2 $2^{+}$ILC2s in the pulmonary tract of the indicated mice after treatment is shown. (B, C) Data are shown as mean + SEM of $n=2-4$ mice per group and are representative of two independent experiments. ${ }^{*} p<0.05,{ }^{* *} p<0.01,{ }^{* * *} p<0.001$, one-way ANOVA analysis followed by Turkey post-tests to analyze statistical significance between PBS control versus treated animals. ns: not significant.

population of IL-5+ and $\mathrm{IL}-13^{+}$cells in the BAL was in the same range as found for Th2 cells.

\section{ILC2 are major IL-5 and IL-13 producers in ovA-induced asthma}

Finally, we determined the contribution of ILC2 to cytokine production in an OVA-induced asthma model. After i.v. injection of Th2-polarized $\mathrm{T}$ cells bearing the DO11.10 OVA-specific T-cell receptor [35], mice were challenged five times with OVA aerosols and analyzed 2 days after the last challenge. In this asthma model, total BAL cellularity increased to $3.0 \pm 0.5 \times 10^{6}$ cells and flow cytometric analysis revealed that $68 \pm 2 \%$ of BAL cells were eosinophils (data not shown), confirming induction of eosinophilic airway inflammation. Flow cytometric analyses of the lungs revealed that ILC2s contained substantial proportions $\mathrm{IL}_{-} 5^{+}$and
IL- $13^{+}$cells, while only small proportions of the cells were IL-4 ${ }^{+}$ (Fig. 6A).

When we determined the contribution of ILC2s to Th2 cytokine production, we noticed that ILC2s were poor producers of IL-4 in both lung and BAL, but were major producers of IL-5. In the lung $\sim 21,000$ ILC2s produced IL-5 (compared with $\sim 32,000 \mathrm{~T} 1 / \mathrm{ST}^{+}$ Th2 cells), while in BAL ILC2 was the main IL-5-producing cell population: $\sim 7500$ ILC2s, compared with $\sim 1900 \mathrm{~T} 1 / \mathrm{ST}^{+}$Th2 cells (Fig. $6 \mathrm{~B}$ ). In addition, we found $\sim 12,000 \mathrm{IL}-13$ producing ILC2 in the lung, compared to $\sim 16,000$ IL-13 producing T1/ST2 ${ }^{+}$ Th2 cells. In contrast, the contribution of ILC2s to IL-13 production in the BAL was limited.

In summary, these results indicate an important role for pulmonary ILC2s in the production of IL-5 and IL-13 in OVA-induced asthma. In particular, pulmonary ILC2s are potent producers of IL-5 and make up the largest IL-5-producing population in the BAL fluid in OVA-induced asthma. 
A
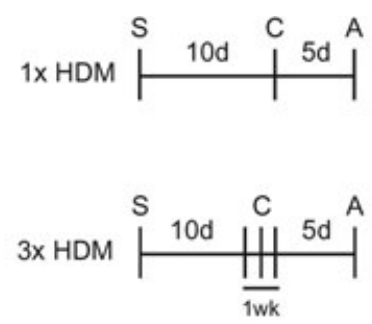

B

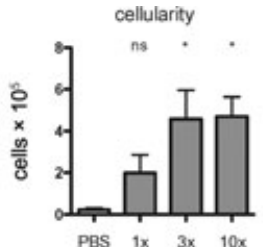

C

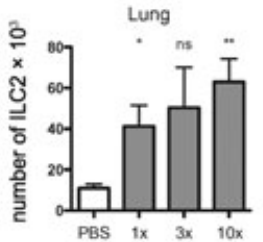

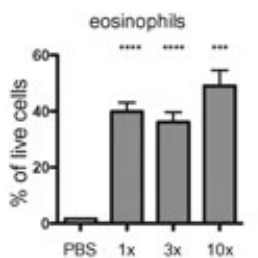
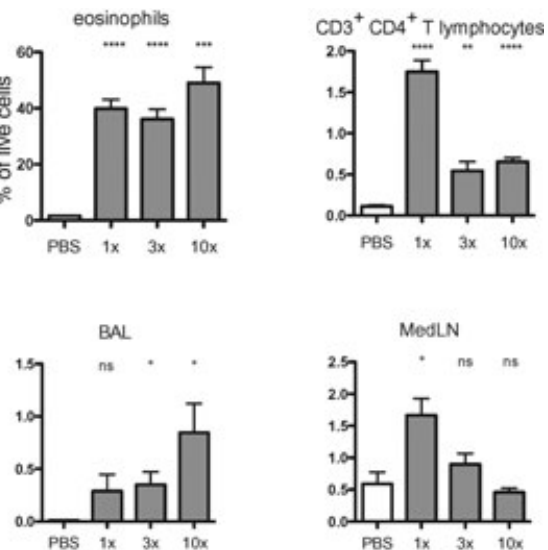

D
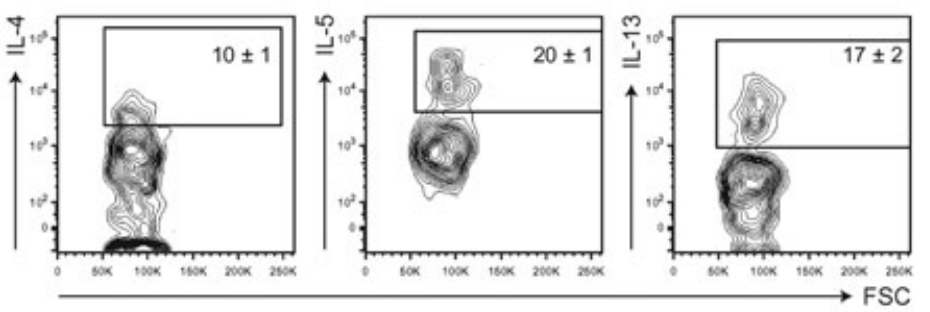

E
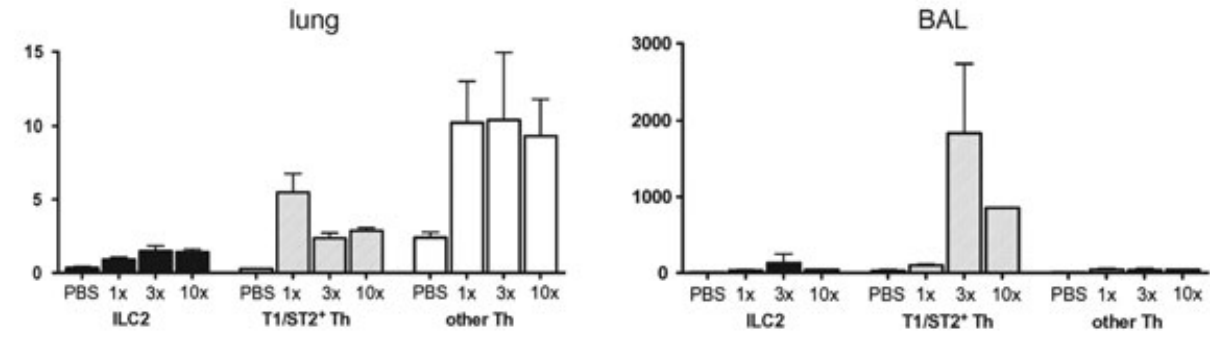

IL-4
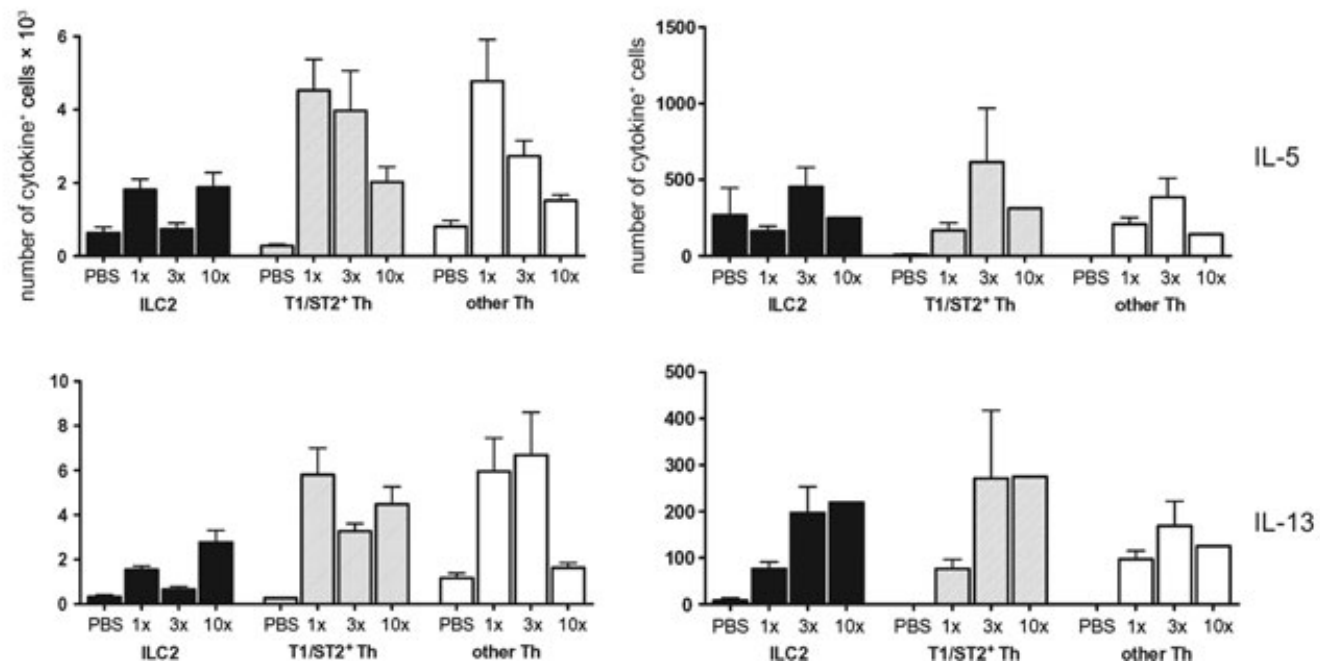

Figure 5. Pulmonary ILC2s are major Th2 cytokine producers in HDM asthma. (A) Asthma protocols showing sensitization (S) and challenges (C) with house dust mite (HDM) in BALB/c mice, followed by analysis (A). Control mice were treated with PBS instead of HDM. (B) Flow cytometric quantification of the indicated cell populations in the BAL fluid after various HDM-asthma treatments is shown. (C) Quantification of Lin $^{-} \mathrm{CD} 127^{+} \mathrm{T} 1 / \mathrm{ST} 2^{+}$ILC2 numbers in lung, BAL, and mediastinal lymph nodes (MedLNs) of HDM- or PBS-treated animals is shown. (D) The intracellular cytokine content in HDM-challenged mice is shown. Total lung cell suspensions were stimulated with PMA/ionomycin for $4 \mathrm{~h}$. $\mathrm{CD} 3^{-} \mathrm{CD} 4^{-} \mathrm{T} 1 \mathrm{ST} 2^{+} \mathrm{CD} 127^{+}$cells were gated and analyzed for the indicated cytokines. Values indicate the proportions of cytokine positive cells \pm SEM. (E) Cytokine production by lymphocyte populations in lung and BAL fluid is shown. Bars show the absolute numbers of intracellular cytokine-positive cells within the indicated lymphocyte populations. (B, C, E) Data are shown as mean + SEM of $n>4$ mice per group and are representative of three experiments. ${ }^{*} p<0.05,{ }^{* *} p<0.01,{ }^{* * *} p<0.001,{ }^{* * * *} p<0.0001$, unpaired t-test. ns: not significant. 
A

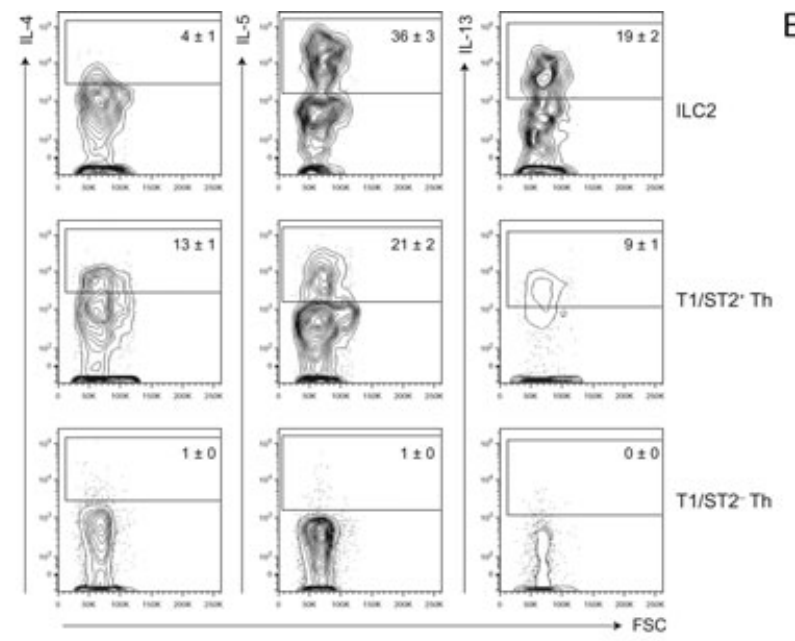

B

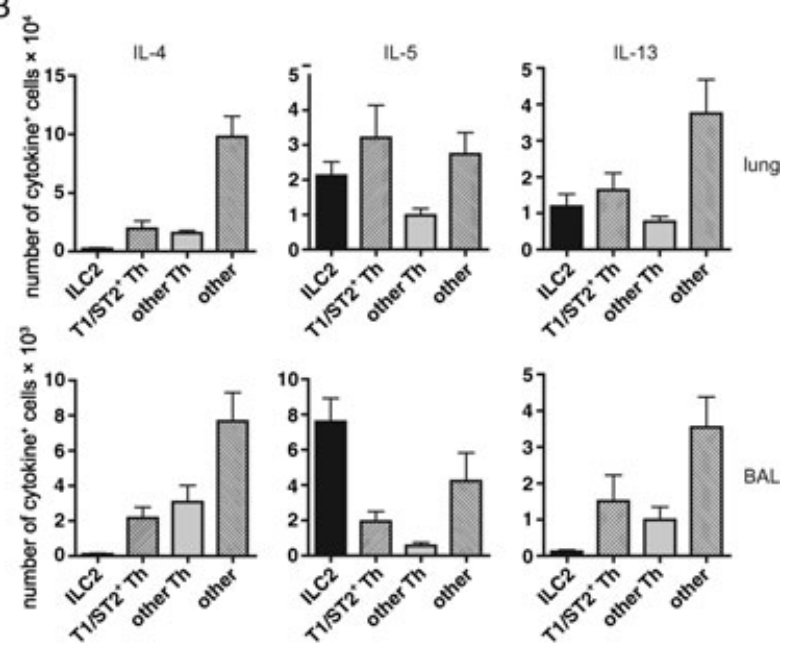

Figure 6. Pulmonary ILC2s are major Th2 cytokine producers in OVA-induced asthma. (A) Flow-cytometric analysis of intracellular cytokine content in OVA-challenged mice is shown. Total lung cell suspensions were stimulated with PMA/ionomycin for $4 \mathrm{~h}$. ILC2s were gated as $\mathrm{Lin}^{-} \mathrm{CD} 25^{+} \mathrm{CD} 127^{+} \mathrm{T} 1 / \mathrm{ST} 2^{+}$. Th cells were gated as $\mathrm{T} 1 / \mathrm{ST} 2^{+}$or $\mathrm{T} 1 / \mathrm{ST} 2^{-} \mathrm{CD} 3^{+} \mathrm{CD}^{+}$lymphocytes. Values indicate the proportions cells $\pm \mathrm{SEM}$ positive for the indicated cytokines. Plots are from representative samples of seven animals per group. (B) Contribution of lymphocyte populations indicated in panel A to production of IL-4 and IL-5, IL-13 in lungs, and BAL fluid, upon OVA treatment in vivo. Data are shown as mean + SEM of $n=7$ animals. Experiment was performed once.

\section{Discussion}

ILC2 were shown to mediate airway hyperreactivity, independent of the adaptive immune system, in a nonallergic form of asthma induced by influenza virus infection. However, whether ILC2 have the capacity to synergize with allergen-specific Th2 cells in allergic asthma remained unexplored. Our studies in naive mice as well as in cytokine-, HDM-, and OVA-induced asthma demonstrate the presence of ILC2s in the pulmonary tract and their substantial contribution to allergic inflammation. We conclude that ILC2s, defined as non-B/non-T lymphocytes expressing IL-7R $\alpha, \mathrm{CD} 25$, Sca-1, c-Kit, and T1/ST2/IL-33R are major producers of IL-5 and IL-13, even in allergen-induced asthma, where Th2 cells are thought to be the most important sources of IL-5 and IL-13.

It was previously reported that the lineage-negative cells that produce IL-5 and IL-13 in response to intranasal administration of IL-25 were T-cell independent, because they were also detected in $R a g 2^{-/-}$mice [14]. We have now shown that these cells are ILC2s and we additionally found that they were efficiently induced upon IL-25 or IL-33 administration in Rag2 $2^{-/-}$mice, but not in Rag2/Il2gc double-deficient mice. While our studies were in progress, two other studies have demonstrated the activation of ILC2s in response to IL-25 and IL-33 administration [36] or in response to glycolipid antigens, which activate NKT cells and thereby induce IL-33 production by alveolar macrophages [37], although these papers focus on the production of IL-13 by ILC2s. From our findings, we can conclude that ILC2s are an important source of both IL-5 and IL-13, not only in IL-25- or IL-33induced allergic lung inflammation, but also in models of allergic asthma based on OVA and HDM allergens. In addition, we noticed that upon IL-33 stimulation in vivo (Fig. 3A) or in
OVA- or HDM-induced airway inflammation (Fig 5D and 6A) low but detectable proportions of ILC2 had the capacity to produce IL-4. We have compared the expression levels of CD127/IL-7R $\alpha$, IL-2R $\alpha, T 1 / S T 2$, and Sca-1 by flow cytometry in IL-4, IL-5, and IL-13 expressing ILC2, but did not detect significant differences.

ILC2s express c-Kit, IL-2R $\alpha$, and IL-7R $\alpha$, suggesting a lymphoid origin. Although the exact nature of ILC2 precursors remains unknown, recent data confirm that bone marrow lymphoid-primed multipotent progenitors and common lymphoid progenitors but not myeloid-erythryoid precursors can give rise to ILC2s [38]. Like other ILC subtypes that have recently been described (reviewed in [6]) and NK cells, ILC2s depend on the transcription factor Id2 [7, 39, 40]. The absence of ILC2s in Rag2/Il2rg doubledeficient mice demonstrates dependency on a $\gamma_{c}$-chain cytokine. More specifically, ILC2s found in FALCs were found to be dependent on IL-7 for their development [7]. We observed pulmonary ILC2s in mice lacking Il2rb (the $\beta$-chain of the IL-2R/IL-15R), showing that IL-2 and IL-15 are not crucial for their development and maintenance, or that their function can be compensated for by other cytokines (R.K.W., Vosshenrich et al., unpublished findings). It is possible that the ILC2 populations that have been recently described share a common precursor and that the local environment plays a decisive role in their terminal differentiation.

In summary, we have shown that the novel population of ILC2 surprisingly constitutes an important fraction of IL-5 and IL-13 cytokine producing cells, next to the classical Th2 cells, in HDM or OVA allergen-induced allergic asthma in mice. It is conceivable that ILC2s also play a crucial role in "classical" allergic asthma in human, because recent genome-wide association studies have demonstrated associations with variations in genes coding for IL-33 and its receptor ST2 [41]. ILC2s could therefore be 
an important novel target for development of future asthma therapies.

\section{Materials and methods}

\section{Mice and asthma induction}

C57BL/ 6 and BALB/c mice were purchased from Harlan. All other mice were bred and kept at the Erasmus MC Laboratory Animal Center. Mice were kept under specific pathogen-free conditions and provided with food and water ad libitum. Mice were 6-15 weeks at time of analysis.

In the cytokine-induced asthma models, 6-week-old anesthetized BALB/c mice were intranasally treated three (IL-25) or five (IL-33 and PBS) times, with $1 \mu \mathrm{g}$ recombinant mouse IL-25 or IL-33 (both R\&D Systems) in $50 \mu \mathrm{L}$ PBS or PBS alone. Mice were sacrificed and analyzed $24 \mathrm{~h}$ later.

For the HDM asthma protocol, 6-week-old BALB/c mice were anesthetized using isoflurane and sensitized intratracheally using $100 \mu \mathrm{g}$ HDM (Greer, Lenoir, NC, USA) or PBS (GIBCO, Invitrogen). Ten days later, mice were challenged once, three, or ten times (three times per week) with $10 \mu \mathrm{g}$ HDM or PBS intranasally. Five days after the last challenge, mice were sacrificed for analysis. OVA asthma was induced by transfer of polarized Th2 cells bearing an OVA-specific TCR as described previously [42]. In brief, D011.10 cells obtained from lymph nodes of naive mice were stimulated in vitro for 6 days in the presence of IL-4 $(10 \mathrm{ng} / \mathrm{mL}$, PeproTech), anti-IFN- $\gamma$ (10 $\mu \mathrm{g} / \mathrm{mL}$, Bioceros B.V., The Netherlands), anti-IL-12 (1 $\mu \mathrm{g} / \mathrm{mL})$, and OVA peptide $(5 \mu \mathrm{g} / \mathrm{mL}$, Worthington Biochemical Corp). Th2 differentiation was confirmed by flow cytometric analysis of intracellular contents for IFN- $\gamma$, IL-4, IL-5, IL-13, and IL-17 production. After washing, $3 \times 10^{6}$ cells were injected into naive BALB/c mice. Mice were challenged with daily OVA aerosols (Sigma-Aldrich) for the subsequent 5 days and sacrificed $48 \mathrm{~h}$ after the last challenge.

BAL fluid was obtained by flushing the lungs three times with $1 \mathrm{~mL}$ PBS containing $0.5 \mathrm{mM}$ EDTA. All experiments were approved by the Erasmus MC Animal Ethics Committee (DEC).

\section{Preparation of single-cell suspensions}

Single-cell suspensions were prepared from the spleen, lungs, MedLNs, and BAL fluid. Spleen and lymph nodes were mechanically disrupted using a $100 \mu \mathrm{m}$ cell strainer (BD Falcon). Lungs were mechanically disrupted using a cell strainer or digested using collagenase type I or collagenase IV, according to the manufacturer's protocol. Mesenteric and thoracic fat was digested using $2 \mathrm{mg} / \mathrm{mL}$ collagenase I in DMEM supplemented with 4\% FCS (Lonza) rocking at $37^{\circ} \mathrm{C}$ for $1 \mathrm{~h}$. Red blood cells from lung and spleen were lysed using osmotic lysis buffer $\left(\mathrm{NH}_{4} \mathrm{Cl}+\mathrm{KHCO}_{3}+\right.$ $\mathrm{Na}_{2}$ EDTA in $\mathrm{H}_{2} \mathrm{O}$ ).

\section{Th cell cultures}

For in vitro T-cell stimulations and Th1/Th2 polarization cultures, naive $\mathrm{CD}_{2} \mathrm{~L}^{+} \mathrm{CD} 4^{+} \mathrm{T}$ cells were purified by cell sorting using a FACSAria equipped with BD FACSDiva software (BD Biosciences) and polarized in vitro as described previously [43]. The purity of obtained fractions was $>98 \%$.

\section{Cell sorting, RNA extraction, and PCR}

FALC cells, BAL, and lung ILC2 were purified by cell sorting using a FACSAria. Fifty cells per well were dispensed directly into 96-well plates and were stimulated for $4 \mathrm{~h}$ using IL-2, IL-25, and IL-33 in the presence of GolgiStop. Polarized Th subsets were also dispensed in 96-wells plates using a FACSAria. After stimulation, cells were directly lysed, resuspended, and frozen until analysis in SuperScript III One-Step RT-PCR reaction buffer containing Igepal CA-630 (Sigma) and RNase Out. Multiplex PCR was performed to directly amplify mRNA using primers for Il4, Il5, Il6, Il13, and Actb. mRNA levels were quantified in a nested RT-PCR reaction using Maxima ${ }^{\mathrm{TM}}$ SYBR Green/ROX qPCR Master Mix (Fermentas) and gene-specific primers. Quantitative real-time PCR was performed using an Applied Biosystems Prism 7700 sequence detection system. The expression of Il5 was calculated by substraction of Actb levels from the test mRNA, followed by calculation of the inverse $\log _{2}$ of the difference. Primers were ordered from Invitrogen and sequences were as follows for external primers: Il5-extF GTGGGGGTACTGTGGAAATG, Il5-extR ACCAAGGAACTCTTGCAGGT, Il4-extF ACGGATGCGACAAAAATCAC, Il4-extR TTGGAAGCCCTACAGACGAG, I16-extF GATGGATGCTACCAAACTGGA, I16-extR TGAAGGACTCTGGCTTTGTCT, I113-extF CCATCTACAGGACCCAGAGG, Il13-extR GCGAAACAGTTGCTTTGTGT, Actb-extF CCAACCGTGAAAAGATGACC, Actb-extR TCTCCGGAGTCCATCACAAT. Internal primers were as follows: ActbF TCAACACCCCAGCCATGTA, ActbR GTGGTACGACCAGAGGCATAC, Il5-forward ACATTGACCGCCAAAAAGAG, I15-reverse ATCCAGGAACTGCCTCGTC.

\section{Flow cytometric analysis}

Cells were stained with a mix of fluorescently tagged antibodies in FACS buffer containing BSA. For measurements of cytokine production by flow cytometry, cells were restimulated for $4 \mathrm{~h}$ at $37^{\circ} \mathrm{C}$ using PMA and ionomycin supplemented with GolgiStop. After extracellular staining, cells were fixed using 4\% PFA and permeabilized using $0.5 \%$ saponin in FACS buffer. Lineagenegative cells were gated as cells not expressing CD3, CD4, CD8 $\alpha$, CD11b, CD11c, CD19, B220, NK1.1, FceRI $\alpha$, Ter-119, and Gr-1. Purchased antibodies are described in Supporting Information Table 1. Biotin-conjugated antibodies were detected using Pacific Blue or allophycocyanin-Cy7-conjugated (BD) streptavidin. Data acquisition was done using a LSR II flow cytometer equipped with three lasers and FACSDiva ${ }^{\mathrm{TM}}$ software. Data analysis was done using FlowJo 8.8.7 (Tree Star Inc., Ashland, OR, USA). 


\section{Statistical analysis}

For comparisons between two groups either unpaired $t$-tests (normal distribution) or Mann-Whitney U tests (if no normal distribution was expected) were used. Statistical significance of differences between more than two groups was calculated using one-way ANOVA analysis followed by Turkey post-tests. All analyses were performed using Prism (GraphPad software, La Jolla, CA, USA).

Acknowledgements: We thank Bart Lambrecht, Mirjam Kool, Tom Cupedo and Ferry Cornelissen (Erasmus MC Rotterdam) and James Di Santo (Institut Pasteur, Paris) for valuable discussions regarding this research. We thank Björn Clausen, Christian Wohn, Tom Cupedo, Ferry Cornelissen, Kim van der Weerd, Odilia Corneth and Erik Lubberts for providing various mouse strains. RKW is supported by a scholarship from the Ligue Nationale Contre le Cancer (LNCC), France.

Conflict of interest: The authors declare no financial or commercial conflict of interest.

\section{References}

1 Lambrecht, B. N. and Hammad, H., Biology of lung dendritic cells at the origin of asthma. Immunity 2009. 31: 412-424.

2 Kim, H. Y., DeKruyff, R. H. and Umetsu, D. T., The many paths to asthma: phenotype shaped by innate and adaptive immunity. Nat. Immunol. 2010. 11: $577-584$.

3 Robinson, D. S., Hamid, Q., Ying, S., Tsicopoulos, A., Barkans, J., Bentley, A. M., Corrigan, C. et al., Predominant TH2-like bronchoalveolar T-lymphocyte population in atopic asthma. N. Engl. J. Med. 1992. 326: 298-304.

4 Holgate, S. T. and Polosa, R., Treatment strategies for allergy and asthma. Nat. Rev. Immunol. 2008. 8: 218-230.

5 Reinhardt, R. L., Liang, H. E. and Locksley, R. M., Cytokine-secreting follicular $\mathrm{T}$ cells shape the antibody repertoire. Nat. Immunol. 2009. 10: 385-393.

6 Spits, H. and Di Santo, J. P., The expanding family of innate lymphoid cells: regulators and effectors of immunity and tissue remodeling. Nat. Immunol. 2011. 12: 21-27.

7 Moro, K., Yamada, T., Tanabe, M., Takeuchi, T., Ikawa, T., Kawamoto, H., Furusawa, J. et al., Innate production of $\mathrm{T}(\mathrm{H}) 2$ cytokines by adipose tissue-associated c-Kit(+)Sca-1(+) lymphoid cells. Nature 2010. 463: 540544.

8 Saenz, S. A., Siracusa, M. C., Perrigoue, J. G., Spencer, S. P., Urban, J. F., Jr., Tocker, J. E., Budelsky, A. L. et al., IL25 elicits a multipotent progenitor cell population that promotes $\mathrm{T}(\mathrm{H}) 2$ cytokine responses. Nature 2010. 464: 1362-1366.

9 Neill, D. R., Wong, S. H., Bellosi, A., Flynn, R. J., Daly, M., Langford, T. K., Bucks, C. et al., Nuocytes represent a new innate effector leukocyte that mediates type-2 immunity. Nature 2010. 464: 1367-1370.
10 Chang, Y. J., Kim, H. Y., Albacker, L. A., Baumgarth, N., McKenzie, A. N., Smith, D. E., Dekruyff, R. H. et al., Innate lymphoid cells mediate influenza-induced airway hyper-reactivity independently of adaptive immunity. Nat. Immunol. 2011. 12: 631-638.

11 Mjosberg, J. M., Trifari, S., Crellin, N. K., Peters, C. P., van Drunen, C. M., Piet, B., Fokkens, W. J. et al., Human IL-25- and IL-33-responsive type 2 innate lymphoid cells are defined by expression of CRTH2 and CD161. Nat. Immunol. 2011. 12: 1055-1062.

12 Monticelli, L. A., Sonnenberg, G. F., Abt, M. C., Alenghat, T., Ziegler, C. G., Doering, T. A., Angelosanto, J. M. et al., Innate lymphoid cells promote lung-tissue homeostasis after infection with influenza virus. Nat. Immunol. 2011. 12: 1045-1054.

13 Hammad, H., Chieppa, M., Perros, F., Willart, M. A., Germain, R. N. and Lambrecht, B. N., House dust mite allergen induces asthma via Toll-like receptor 4 triggering of airway structural cells. Nat. Med. 2009. 15: 410416.

14 Hurst, S. D., Muchamuel, T., Gorman, D. M., Gilbert, J. M., Clifford, T., Kwan, S., Menon, S. et al., New IL-17 family members promote Th1 or Th2 responses in the lung: in vivo function of the novel cytokine IL-25. J. Immunol. 2002. 169: 443-453.

15 Fort, M. M., Cheung, J., Yen, D., Li, J., Zurawski, S. M., Lo, S., Menon, S. et al., IL-25 induces IL-4, IL-5, and IL-13 and Th2-associated pathologies in vivo. Immunity 2001. 15: 985-995.

16 Dolgachev, V., Petersen, B. C., Budelsky, A. L., Berlin, A. A. and Lukacs, N. W., Pulmonary IL-17E (IL-25) production and IL-17RB+ myeloid cellderived Th2 cytokine production are dependent upon stem cell factorinduced responses during chronic allergic pulmonary disease. J. Immunol. 2009. 183: 5705-5715.

17 Tamachi, T., Maezawa, Y., Ikeda, K., Kagami, S., Hatano, M., Seto, Y., Suto, A. et al., IL-25 enhances allergic airway inflammation by amplifying a TH2 cell-dependent pathway in mice. J. Allergy Clin. Immunol. 2006. 118: 606-614.

18 Schmitz, J., Owyang, A., Oldham, E., Song, Y., Murphy, E., McClanahan, T. K., Zurawski, G. et al., IL-33, an interleukin-1-like cytokine that signals via the IL-1 receptor-related protein ST2 and induces T helper type 2associated cytokines. Immunity 2005. 23: 479-490.

19 Coyle, A. J., Lloyd, C., Tian, J., Nguyen, T., Erikkson, C., Wang, L., Ottoson, P. et al., Crucial role of the interleukin 1 receptor family member T1/ST2 in T helper cell type 2-mediated lung mucosal immune responses. J. Exp. Med. 1999. 190: 895-902.

20 Lohning, M., Stroehmann, A., Coyle, A. J., Grogan, J. L., Lin, S., GutierrezRamos, J. C., Levinson, D. et al., T1/ST2 is preferentially expressed on murine Th2 cells, independent of interleukin 4, interleukin 5, and interleukin 10, and important for Th2 effector function. Proc. Natl. Acad. Sci. USA 1998. 95: 6930-6935.

21 Prefontaine, D., Nadigel, J., Chouiali, F., Audusseau, S., Semlali, A., Chakir, J., Martin, J. G. et al., Increased IL-33 expression by epithelial cells in bronchial asthma. J. Allergy Clin. Immunol. 2010. 125: 752-754.

22 Ballantyne, S. J., Barlow, J. L., Jolin, H. E., Nath, P., Williams, A. S., Chung, K. F., Sturton, G. et al., Blocking IL-25 prevents airway hyperresponsiveness in allergic asthma. J. Allergy Clin. Immunol. 2007. 120: 13241331.

23 Kearley, J., Buckland, K. F., Mathie, S. A. and Lloyd, C. M., Resolution of allergic inflammation and airway hyperreactivity is dependent upon disruption of the T1/ST2-IL-33 pathway. Am. J. Respir. Crit. Care Med. 2009. 179: 772-781.

24 Price, A. E., Liang, H. E., Sullivan, B. M., Reinhardt, R. L., Eisley, C. J., Erle, D. J. and Locksley, R. M., Systemically dispersed innate IL-13-expressing cells in type 2 immunity. Proc. Natl. Acad. Sci. USA 2010. 107: 11489-11494. 
25 Kondo, Y., Yoshimoto, T., Yasuda, K., Futatsugi-Yumikura, S., Morimoto, M., Hayashi, N., Hoshino, T. et al., Administration of IL-33 induces airway hyperresponsiveness and goblet cell hyperplasia in the lungs in the absence of adaptive immune system. Int. Immunol. 2008. 20: 791-800.

26 Kurowska-Stolarska, M., Kewin, P., Murphy, G., Russo, R. C., Stolarski, B., Garcia, C. C., Komai-Koma, M. et al., IL-33 induces antigen-specific IL-5+ $\mathrm{T}$ cells and promotes allergic-induced airway inflammation independent of IL-4. J. Immunol. 2008. 181: 4780-4790.

27 Kroeger, K. M., Sullivan, B. M. and Locksley, R. M., IL-18 and IL-33 elicit Th2 cytokines from basophils via a MyD88- and p38alpha-dependent pathway. J. Leukoc. Biol. 2009. 86: 769-778.

28 Zheng, Y., Valdez, P. A., Danilenko, D. M., Hu, Y., Sa, S. M., Gong, Q., Abbas, A. R. et al., Interleukin-22 mediates early host defense against attaching and effacing bacterial pathogens. Nat. Med. 2008. 14: 282289.

29 Buonocore, S., Ahern, P. P., Uhlig, H. H., Ivanov, II, Littman, D. R., Maloy, K. J. and Powrie, F., Innate lymphoid cells drive interleukin23-dependent innate intestinal pathology. Nature 2010. 464: $1371-$ 1375.

30 Cella, M., Fuchs, A., Vermi, W., Facchetti, F., Otero, K., Lennerz, J. K., Doherty, J. M. et al., A human natural killer cell subset provides an innate source of IL-22 for mucosal immunity. Nature 2009. 457: 722725 .

31 Luci, C., Reynders, A., Ivanov, II, Cognet, C., Chiche, L., Chasson, L., Hardwigsen, J. et al., Influence of the transcription factor RORgammat on the development of NKp46+ cell populations in gut and skin. Nat. Immunol. 2009. 10: 75-82.

32 Sanos, S. L., Bui, V. L., Mortha, A., Oberle, K., Heners, C., Johner, C. and Diefenbach, A., RORgammat and commensal microflora are required for the differentiation of mucosal interleukin 22-producing NKp46+ cells. Nat. Immunol. 2009. 10: 83-91.

33 Satoh-Takayama, N., Vosshenrich, C. A., Lesjean-Pottier, S., Sawa, S., Lochner, M., Rattis, F., Mention, J. J. et al., Microbial flora drives interleukin 22 production in intestinal NKp46+ cells that provide innate mucosal immune defense. Immunity 2008. 29: 958-970.

34 Sharkhuu, T., Matthaei, K. I., Forbes, E., Mahalingam, S., Hogan, S. P., Hansbro, P. M. and Foster, P. S., Mechanism of interleukin-25 (IL-17E)induced pulmonary inflammation and airways hyper-reactivity. Clin. Exp. Allergy 2006. 36: 1575-1583.

35 Murphy, K. M., Heimberger, A. B. and Loh, D. Y., Induction by antigen of intrathymic apoptosis of CD4+CD8+TCRlo thymocytes in vivo. Science 1990. 250: 1720-1723.

36 Barlow, J. L., Bellosi, A., Hardman, C. S., Drynan, L. F., Wong, S. H., Cruickshank, J. P. and McKenzie, A. N., Innate IL-13-producing nuocytes arise during allergic lung inflammation and contribute to airways hyperreactivity. J. Allergy Clin. Immunol. 2012. 129: 191-198.

37 Kim, H. Y., Chang, Y. J., Subramanian, S., Lee, H. H., Albacker, L. A. Matangkasombut, P., Savage, P. B. et al., Innate lymphoid cells responding to IL-33 mediate airway hyperreactivity independently of adaptive immunity. J. Allergy Clin. Immunol. 2012. 129: 216-227.

38 Yang, Q., Saenz, S. A., Zlotoff, D. A., Artis, D. and Bhandoola, A., Cutting edge: natural helper cells derive from lymphoid progenitors. J. Immunol. 2011. 187: 5505-5509.

39 Yokota, Y., Mansouri, A., Mori, S., Sugawara, S., Adachi, S., Nishikawa, S. and Gruss, P., Development of peripheral lymphoid organs and natural killer cells depends on the helix-loop-helix inhibitor Id2. Nature 1999. 397: 702-706.

40 Satoh-Takayama, N., Lesjean-Pottier, S., Vieira, P., Sawa, S., Eberl, G., Vosshenrich, C. A. and Di Santo, J. P., IL-7 and IL-15 independently program the differentiation of intestinal CD3-NKp46+ cell subsets from Id2dependent precursors. J. Exp. Med. 2010. 207: 273-280.

41 Ober, C. and Yao, T. C., The genetics of asthma and allergic disease: a 21st century perspective. Immunol. Rev. 2011. 242: 10-30.

42 van Rijt, L. S., Jung, S., Kleinjan, A., Vos, N., Willart, M., Duez, C., Hoogsteden, H. C. et al., In vivo depletion of lung CD11c + dendritic cells during allergen challenge abrogates the characteristic features of asthma. J. Exp. Med. 2005. 201: 981-991.

43 Ribeiro de Almeida, C., Heath, H., Krpic, S., Dingjan, G. M., van Hamburg, J. P., Bergen, I., van de Nobelen, S. et al., Critical role for the transcription regulator CCCTC-binding factor in the control of Th2 cytokine expression. J. Immunol. 2009. 182: 999-1010.

Abbreviations: BAL: broncho-alveolar lavage - FALC: fat-associated lymphoid cluster $\cdot \gamma$ c: common gamma $\cdot$ HDM: house dust mite $\cdot$ ILC: innate lymphoid cell. MedLN: mediastinal lymph node

Full correspondence: Dr. Rudi W. Hendriks, Department of Pulmonary Medicine, Room Ee2251a. Erasmus MC Rotterdam. PO Box 2040 NL 3000 CA. Rotterdam. The Netherlands

Fax: +31-10-7044728

e-mail: r.hendriks@erasmusmc.nl

See accompanying Commentry: http://dx.doi.org/10.1002/eji.

201242549

Received: 9/8/2011

Revised: 20/12/2011

Accepted: 23/1/2012 